\title{
Heuristics for Production Allocation and Ordering Policies in Multi-Plants with Capacity
}

\author{
Jie Zang ${ }^{1,2}$, Jiafu Tang ${ }^{1}$ \\ ${ }^{1}$ School of Information Science and Engineering, Northeastern University, Shenyang, China; ${ }^{2}$ School of Information, Liaoning \\ University, Shenyang, China. \\ Email: jiez00509@sina.com, jftang@mail.neu.edu.cn
}

Received October $19^{\text {th }}$, 2009; revised November 27 ${ }^{\text {th }}$, 2009; accepted January $5^{\text {th }}, 2010$.

\begin{abstract}
Joint decisions in production allocation and ordering policies for single and multiple products in a production-distribution network system consisting of multiple plants are discussed, production capacity constraints of multi-plants and unit production capacity for producing a product are considered. Based on the average total cost in unit time, the decisive model is established. It tries to determine the production cycle length, delivery frequency in a cycle from the warehouse to the retailer and the economic production allocation. The approach hinges on providing an optimized solution to the joint decision model through the heuristics methods. The heuristic algorithms are proposed to solve the single-product joint decision model and the multi-products decision problem. Simulations on different sizes of problems have shown that the heuristics is effective, and in general more effective than Quasi-Newton method (QNM).
\end{abstract}

Keywords: Joint Decisions, Production-Distribution; Multiple Plants, Capacitated, Heuristic Algorithm

\section{Introduction}

In the past, logistic decision among material procurement management, production and distribution were made in isolation. Previous studies have examined production, transportation and inventory separately. These major activities are closely related with each other and should be coordinated effectively to enhance its profit in today's competitive market. Uncoordinated and isolated decision-making among functional related activities in supply chain system may weaken its system-wide competitiveness. Hence, more efforts are now being made to integrate coordinate production and distribution, production and transportation, production and inventory, as well as transportation and inventory in the form of supply chain management.

King [1] described the implementation of a coordinated production-distribution system, a major tire manufacturer with four factories and nine major distribution centers. Williams [2] considered the problem of joint scheduling of production and distribution in a complex network, the objective of the problem was to minimize average production and distribution cost per period. Hill [3] discussed production-delivery policies in a single manufacturer and a single retailer. David [4] attempted to identify lot sizing and delivery schedul- ing in a single manufacturer and a single retailer system. Kim [5] discussed the production and ordering policies in a supply chain consisting of a single manufacturer and a single retailer. He proposes an efficient heuristic algorithm to determine the near optimal production allocation ratios. Kim [6] extended their paper and develops joint economic production allocation, lot-sizing, and shipment policies in a supply chain where a manufacturer produces multiple items in multiple production lines and ships the items to the respective retailers. Their formulations are often based on economic order quantity (EOQ) and mathe- matical programming. Accordingly, the corresponding solution methods are EOQ [7,8], heuristics [5,6,9] and decomposition $[10,11]$.

In recent studies, model for coordinating productiondistribution network systems have tended to focus on joint decisions on all activities. More complicated integrated decisions on production, transportation, and inventory have received relatively little attention, as in [12] and [13]. Tang [12] discussed an integrated decision on production assignment, lot-sizing, transportation, and order quantity for a multiple-supplier/multiple-destinations logistics network in a global manufacturing system and proposed a heuristics to solve medium and largescale integrated decision problems. Yung [13] attempted 
to tackle joint decisions in assigning production, lot-size, transportation, and order quantity for sing and multiple products in a production-distribution network system with multiple suppliers and multiple destinations. He provided an optimized solution to solve the joint decision model through a two-layer decomposition method that combines several heuristics.

This paper addresses the issue of how to effectively allocate production requirement to multiple plants in supply chain system. Kim [5,6] discussed the production and ordering policies in a supply chain consisting of a single manufacturer with multiple plants and a single retailer or multiple retailers. The retailers place orders based on the EOQ-like policy, and the multiple plants produce demand requirement from the retailers. Each of multiple plants has its production and transfer rates. In real life, all the plants in the manufacturer have production capacity constraints. All the plants should produce within its capacity to meet the demands of the retailers. The problem discussed in this paper extends the model proposed by $[5,6]$, and production capacity constraints of multi-plants and unit production capacity for producing a product are considered in the model. The heuristics methods have been developed to solve the problem with single product and multiple products, respectively.

In this paper, the model for a single product will be discussed in Section 2, followed by detailed discussion to solve multiple products in Section 3. One illustrated example with several testing problems and their respective simulation results and analyses are presented in Section 4.

\section{Formulations and Heuristics with Single Product}

\subsection{Problem Formulations}

In a global manufacturing enterprise, there are plants each producing multiple parts and multiple assemblies that serve multiple assembly plants in a year, or alternatively, each assembly plant demands multiple parts from many different suppliers. Hence, such a global manufacturing enterprise can be formulated as a combined production-distribution network consisting of multiple suppliers and multiple destinations. In this paper, we consider a production-distribution network composed of a single manufacturer with multiple plants and multiple retailers. The retailers are given annual demand of the product. To meet the annual demands of the product, the manufacturer procures the materials and multi-plants produce within their capacity in the manufacturer. The multi-plants of the manufacturer have their production rate. The finished products are transferred to the common warehouse at the plants' transfer rate. Finally, the warehouse delivers the ordered lots of a fixed size to the retailer periodically. The network is shown in Figure 1. The cost components considered include two parts, the first part is the ordering cost from raw materials, the pro-

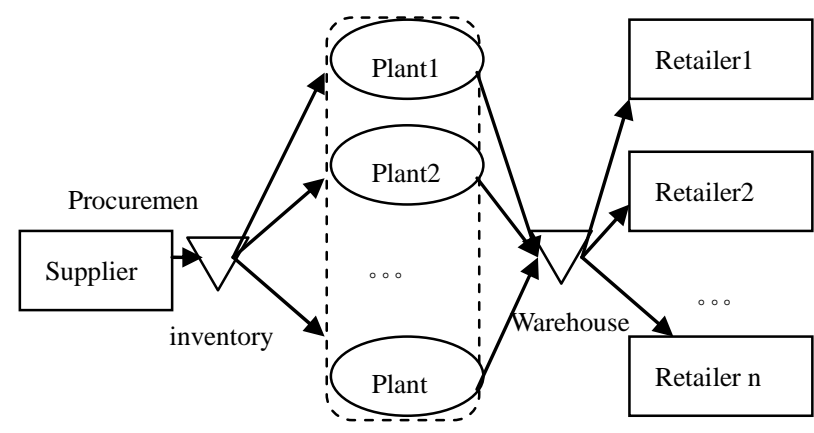

Figure 1. Production-distribution network

duction setup cost, the ordering cost at the warehouse, and the ordering cost of the retailer; the second part is the holding costs for raw materials, work-in-process inventories, finished items at the warehouse and the retailer.

Assume that there are $m$ plants in a manufacturer, where each of the plants is indicated by the subscripts $j$. The following notations and decision variables are applied.

$P_{j}=$ annual production rate at plant $j$ (unit/year)

$Q_{j}=$ annual production capacity at plant $j$ (year)

$d_{j}=$ annual transfer rate from plant $j$ to the warehouse (unit)

$u_{j}=$ production capacity needed to produce unit product at plant $j$ (year)

$h_{j}=$ holding cost for work-in-processes at plant

$S_{p}=$ production setup cost at the manufacturer $(\$)$

$A_{m}=$ ordering cost for raw materials at the manufacturer (\$)

$A_{w}=$ order handling cost for finished products at the warehouse (\$)

$A_{r}=$ ordering cost at the retailer (\$)

$H_{m}=$ holding cost for raw materials at the manufacturer (\$)

$H_{w}=$ holding cost for finished products at the warehouse (\$)

$H_{r}=$ holding cost for finished products at the retailer (\$)

$D=$ demand rate in units at the retailer (unit/year)

$T=$ decision variable, production cycle length at the manufacturer (year)

$m=$ decision variable, delivery frequency in a production cycle from the warehouse to the retailer

$\lambda=\left(\lambda_{1}, \ldots, \lambda_{j}\right)$ decision variable, production allocation for multiple plants

These notations will be extended in Section 3 to include multiple products. Accordingly, from the above parameters and decision variables, $\sum_{j} d_{j} \geq D$ and $P_{j} \geq d_{j}$ should be satisfied for the relevance of the pro- 
posed model.

\subsection{Joint Decision Model for a Single Product}

The average cost components considered in this problem include two parts, the first part is the ordering cost; the second part is the holding costs these two parts of the costs are denoted by $F_{1}(T, m)$ and $F_{2}(m, \lambda)$ respectively. In a production cycle has $m$ delivery from the warehouse to the retailer, so the ordering cost $F_{1}(T, m)$ are given as

$$
F_{1}(T, m)=\left[\left(A_{m}+S_{p}\right)+m\left(A_{w}+A_{r}\right)\right] / T
$$

For the second part of the costs, the average inventory levels for raw materials, work-in-process in plant $(j)$, and finished products at the warehouse and the retailer over the production cycle are denoted by $I_{m}, I_{j}, I_{w}$ and $I_{r}$, respectively. $I_{m}$ and $I_{w}$ can be derived by the appendix of Reference [5]. From the decision variables, we can derived the production lot size is $D T$, and the apportioned production lot size for plant $i$ is $\lambda_{j} D T$. During a production cycle, the production time is $\lambda_{i} D T / P_{i}$, the delivery time is $\lambda_{j} D T / d_{j}$, as illustrated in Figure 2. It can be shown that, the average inventory for work-in-process $I_{j}$ is

$$
\begin{aligned}
I_{j} & =\frac{1}{T}\left[\frac{1}{2}\left(\lambda_{j} D T / d_{j}\right) \lambda_{j} D T-\frac{1}{2}\left(\lambda_{j} D T / P_{j}\right) \lambda_{j} D T\right] \\
& =\left(D^{2} T / 2\right)\left[\left(\lambda_{j}^{2} / d_{j}\right)\left(1-d_{j} / P_{j}\right)\right]
\end{aligned}
$$

Hence, $I_{m}, I_{j}, I_{w}$ and $I_{r}[5]$ are given as

$$
\begin{aligned}
I_{m} & =\left(D^{2} T / 2\right) \sum_{i=1}^{n} \lambda_{j}^{2} / P_{j} \\
I_{j} & =\left(D^{2} T / 2\right)\left[\left(\lambda_{j}^{2} / d_{j}\right)\left(1-d_{j} / P_{j}\right)\right] \\
I_{w} & =(D T / 2)(1+1 / m)-\left(D^{2} T / 2\right) \sum_{i=1}^{n} \lambda_{j}^{2} / d_{j} \\
I_{r} & =D T / 2 m
\end{aligned}
$$

Hence, the holding cost $F_{2}(m, \lambda)$ are given as

$$
F_{2}(m, \lambda)=H_{m} I_{m}+\sum_{j=1}^{n} h_{j} I_{j}+H_{w} I_{w}+H_{r} I_{r}
$$

Substituting (2)-(5) into (6), we can obtain

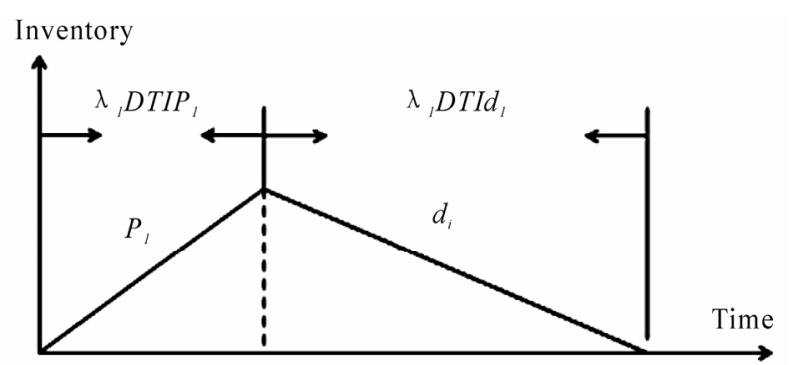

Figure 2. Inventroy trajectory for work-in-process in plant $j$

$$
\begin{aligned}
& F_{2}(m, \lambda)=(D T / 2)\left[H_{w}+\left(H_{w}+H_{r}\right) / m-D \sum_{j=1}^{n} H_{j} \lambda_{j}^{2}\right] \\
& \text { where } \quad \mathrm{H}_{j}=\frac{h_{j}-H_{m}}{P_{j}}+\frac{H_{w}-h_{j}}{d_{j}}
\end{aligned}
$$

The integrated decisions of the economic production allocation and delivery policies are expressed as the following model:

$$
\begin{aligned}
\operatorname{Min} W= & F_{1}(T, m)+F_{2}(m, \lambda) \\
= & {\left[\left(A_{m}+S_{p}\right)+m\left(A_{w}+A_{r}\right)\right] / T } \\
+ & (D T / 2)\left[H_{w}+\left(H_{w}+H_{r}\right) / m-D \sum_{j=1}^{n} H_{j} \lambda_{j}^{2}\right] \\
& \quad \text { s.t. } \sum_{j} \lambda_{j}=1 \\
& 0 \leq \lambda_{j} \leq d_{j} / D \quad \forall j=1,2, \ldots, n \\
& 0 \leq \lambda_{j} \leq Q_{j} / D u_{j} \quad \forall j=1,2, \ldots, n
\end{aligned}
$$

In this model, (8) is the objective of minimizing the average ordering and holding cost for raw materials, work-in-process, finished products at the warehouse and the retailer. The constraint (9) is the allocation vector for multiple plants. The constraints (10) and (11) should be satisfied by definition, respectively.

\subsection{Heuristics Solution Procedures}

The model is a fractional nonlinear programming model that is neither convex nor concave and is difficult to be solved. So we transform this model with the decision variables $(T, m, \lambda)$ into a more simplified and equivalent problem with a decision variable $\lambda$, the last transformed problem is computed using a heuristic procedure.

First, the problem is strictly convex with respect to $T$, thus the optimal cycle length $T^{*}(m, \lambda)$ for a fixed pair of $m$ and $\lambda$ can be uniquely derived by solving $d W / d T=0$ :

$$
\mathrm{T}^{*}=\left\{\frac{2\left[\left(A_{m}+S_{p}\right)+m\left(A_{w}+A_{r}\right)\right]}{\left[H_{w}+\left(H_{w}+H_{r}\right) / m-\sum_{j=1}^{n}\left(D H_{j} \lambda_{j}^{2}\right)\right] D}\right\}^{1 / 2}
$$

Substituting $T^{*}$ into (8), we can derive $\mathrm{E}(\mathrm{m}, \lambda)$ :

$$
\begin{aligned}
& E(m, \lambda)=W\left(T^{*}, m, \lambda\right) \\
& =\left\{2\left[\left(A_{m}+S_{p}\right)+m\left(A_{w}+A_{r}\right)\right]\right. \\
& \left.\left[H_{w}+\left(H_{w}+H_{r}\right) / m-\sum_{j=1}^{n}\left(D H_{j} \lambda_{j}^{2}\right)\right] D\right\}^{1 / 2}
\end{aligned}
$$

For (13), we can derive:

$$
\begin{aligned}
S(m / \lambda)= & {\left[\left(A_{m}+S_{P}\right)+m\left(A_{w}+A_{r}\right)\right] } \\
& {\left[H_{w}+\left(H_{w}+H_{r}\right) / m-\sum_{j=1}^{n}\left(D H_{j} \lambda_{j}^{2}\right)\right] D }
\end{aligned}
$$


We can obtain (15) for fixed $\lambda$ :

$$
\begin{aligned}
\frac{d S(m / \lambda)}{d m}= & \left(A_{w}+A_{r}\right)\left[H_{w}-\sum_{i=1}^{\mathrm{n}} D H_{j} \lambda_{j}^{2}\right] \\
& -\frac{\left(A_{m}+S_{p}\right)\left(H_{w}+H_{r}\right)}{m^{2}} \\
\frac{d^{2} S(m / \lambda)}{d m^{2}} & =\frac{2\left(A_{m}+S_{p}\right)\left(H_{w}+H_{r}\right)}{m^{3}}
\end{aligned}
$$

Since $d^{2} S(m / \lambda) / d m^{2}>0$, we can obtain $m$ from $d S / d m=0$ and is given by

$$
\begin{aligned}
m(\lambda)= & \left\{\left(A_{m}+S_{p}\right)\left(H_{w}+H_{r}\right) /\left(A_{w}+A_{r}\right)\right. \\
& {\left.\left[H_{w}-\sum_{j=1}^{\mathrm{n}}\left(D H_{j} \lambda_{j}^{2}\right)\right]\right\}^{1 / 2} }
\end{aligned}
$$

Since other terms in (17) are constant regardless of $\lambda$ except $\sum_{j=1}^{n} D H_{j} \lambda_{j}^{2}$, we reformulate the next problem equivalently as follows:

$$
\begin{gathered}
\operatorname{Max} G(\lambda)=\sum_{j=1}^{n} H_{j} \lambda_{j}^{2} \\
\text { s.t. (9),(10),(11) }
\end{gathered}
$$

This problem belongs to the class of quadratic maximization problems subject to linear constraints with a positive definite quadratic term. Reference [14] has proved it is an NP-hard problem. Since this problem aims to assign production allocation $\lambda_{j}$, a heuristic procedure is proposed as follows to solve it.

The heuristic algorithm steps

Step1. Resequence $H_{i}$ in the descending order, such that $H_{1} \geq H_{2} \geq H_{3} \geq \cdots \geq H_{m}$;

Step2. Let $t$ be the current index number of the plant to be assigned, and $R_{t}=\sum_{i=1}^{t} \lambda_{i}$ be the total amount of the production allocation $t=0, R_{t}=0$;

Step3. $t=t+1$ assignment to production to the th plant point:

If $R_{t-1}<1$ set

$$
\begin{gathered}
\lambda_{t}=\min \left\{1-R_{t-1}, d_{t} / D, Q_{t} / D u_{t}\right\} \\
R_{t}=R_{t-1}+\lambda_{t}
\end{gathered}
$$

Else $\lambda_{t}=0, R_{t}=R_{t-1}+\lambda_{t}$

End if

Step4. If $t<m$, go to Step 3; else, go to Step5;

Step5. Calculate the $\operatorname{Max} G(\lambda)$, then stop.

After deriving $\lambda *$, we can obtain $m^{*}$ and $T^{*}$ from (16) and (12).

\section{Joint Decisions for Multiple Products}

\subsection{Formulation with Multiple Products}

In many real cases, the manufacture often produces multiple products to meet the need of the retailers. In this pro- duction-distribution network of multiple products, the main issue is how joint decisions can be made annually on production cycle length, delivery frequency and production allocation at a minimal average cost to the network. To derive the solution, the notations are defined as follows:

$P_{i j}=$ annual production rate for product $i$ at plant $j$ (unit/year)

$Q_{i j}=$ annual production capacity for product $i$ at plant $j$ (year)

$d_{i j}=$ annual transfer rate for product $i$ from plant $j$ to the warehouse (unit)

$u_{i j}=$ production capacity needed to produce unit product $i$ at plant $j$ (year)

$h_{i j}=$ holding cost for product $i$ at plant $j(\$)$

$S_{i}=$ production setup cost for product $i$ at the manufacturer (\$)

$A_{i}^{R}=$ ordering cost of raw materials for product $i(\$)$

$A_{i}^{W}=$ order handling cost for finished product $i$ at the warehouse (\$)

$A_{i}^{C}=$ ordering cost for product $i$ at the retailer (\$)

$H_{i}^{R}=$ holding cost of raw materials for product $i(\$)$

$H_{i}^{W}=$ holding cost for finished product $i$ at the warehouse (\$)

$H_{i}^{C}=$ holding cost for finished product $i$ at the retailer (\$)

$D_{i}=$ demand rate for product $i$ (unit/year)

$T=$ decision variable, production cycle length at the manufacturer (year)

$m_{i}=$ decision variable, delivery frequency for product $i$ in a production cycle from the warehouse to the retailer

$\lambda_{i j}=$ decision variable, production allocation for product $i$ in plant $j$

Similar to the average cost structure of a single product, the ordering costs and the holding costs are represented as follows, respectively:

$$
F_{1}\left(T, m_{i}\right)=\sum_{i}\left[\left(A_{i}^{R}+S_{i}\right)+m_{i}\left(A_{i}^{W}+A_{i}^{C}\right)\right] / T
$$

The second part is the holding costs for raw materials, work-in-process inventories, finished items at the warehouse and the retailer. They are denoted by $I_{i}^{R}, I_{i j}$, $I_{i}^{W}, I_{i}^{C}$ respectively

$$
\begin{aligned}
I_{i}^{R} & =\left(D_{i}^{2} T / 2\right) \sum_{j=1}^{n} \lambda_{i j}^{2} / P_{i j} \\
I_{i j} & =\left(D_{i}^{2} T / 2\right) \sum_{j}\left[\left(\lambda_{i j}^{2} / d_{i j}\right)\left(1-d_{i j} / P_{i j}\right)\right] \\
I_{i}^{W} & =\left(D_{i} T / 2\right)\left(1+1 / m_{i}\right)-\left(D_{i}^{2} T / 2\right) \sum_{j=1}^{n} \lambda_{i j}^{2} / d_{i j} \\
I_{i}^{C} & =D_{i} T / 2 m_{i}
\end{aligned}
$$

Hence, the holding cost $F_{2}\left(m_{i}, \lambda_{i j}\right)$ are given as 
$F_{2}\left(m_{i}, \lambda_{i j}\right)=\sum_{i} H_{i}^{R} I_{i}^{R}+\sum_{i j} h_{i j} I_{i j}+\sum_{i} H_{i}^{W} I_{i}^{W}+\sum_{i} H_{i}^{C} I_{i}^{C}$

Substituting (19)-(22) into (23), we can obtain

$$
F_{2}\left(m_{i}, \lambda_{i j}\right)=(T / 2) \sum_{i} D_{i}\left[H_{i}^{W}+\left(H_{i}^{W}+H_{i}^{C}\right) / m_{i}-D_{i} \sum_{j} H_{i j} \lambda_{i j}{ }^{2}\right]
$$

$$
H_{i j}=\left(h_{i j}-H_{i}^{R}\right) / P_{i j}+\left(H_{i}^{W}-h_{i j}\right) / d_{i j}
$$

The integrated decisions of the economic production allocation and delivery policies are expressed as the following model:

$$
\begin{aligned}
& \min F=F_{1}\left(T, m_{i}\right)+F_{2}\left(m_{i}, \lambda_{i j}\right) \\
& =\sum_{i}\left[\left(A_{i}^{R}+S_{i}\right)+m_{i}\left(A_{i}^{W}+A_{i}^{C}\right)\right] / T \\
& +(T / 2) \sum_{i} D_{i}\left[H_{i}^{W}+\left(H_{i}^{W}+H_{i}^{C}\right) / m_{i}-D_{i} \sum_{j} H_{i j} \lambda_{i j}{ }^{2}\right] \\
& \text { s.t } \quad \sum_{j} \lambda_{i j}=1 \quad \forall i \\
& 0 \leq \lambda_{i j} \leq d_{i j} / D_{i} \quad \forall i, j \\
& 0 \leq \lambda_{i j} \leq Q_{i j} / D_{i} u_{i j} \quad \forall j
\end{aligned}
$$

\subsection{Heuristics Method for Multiple Products}

The model is a fractional nonlinear programming model that is the same as the model with the single product. It can be solved by traditional nonlinear programming techniques, such as GINO, gradient search methods, where only the local optimal solution may be found. A heuristics is proposed to solve this problem.

First, the problem is strictly convex with respect to $T$, thus the optimal cycle length $T^{*}\left(m_{i}, \quad \lambda_{i j}\right)$ for a fixed pair of $m_{i}$ and $\lambda_{i j}$ can be uniquely derived by solving $d_{F} / d_{T}=0$ :

$$
T^{*}\left(m_{i}, \lambda_{i j}\right)=\left\{\frac{2 \sum_{i}\left[\left(A_{i}^{R}+S_{i}\right)+m_{i}\left(A_{i}^{W}+A_{i}^{C}\right)\right]}{\sum_{i} D_{i}\left[H_{i}^{W}+\left(H_{i}^{W}+H_{i}^{C}\right) / m_{i}-D_{i} \sum_{j} H_{i j} \lambda_{i j}{ }^{2}\right]}\right\}^{1 / 2}
$$

Substituting $T^{*}$ into (26), we can derive:

$$
\begin{aligned}
& \min F^{\prime}=\left\{2\left[\sum_{i}\left(\left(A_{i}^{R}+S_{i}\right)+m_{i}\left(A_{i}^{W}+A_{i}^{C}\right)\right)\right]\right. \\
& \left.\left[\sum_{i} D_{i}\left(H_{i}^{W}+\left(H_{i}^{W}+H_{i}^{C}\right) / m_{i}-D_{i} \sum_{j} H_{i j} \lambda_{i j}{ }^{2}\right)\right]\right\}^{1 / 2}
\end{aligned}
$$

For (31), we can derive:

$$
\begin{aligned}
& S_{E}\left(m_{i} / \lambda_{i j}\right)=\left\{\sum_{i}\left[\left(A_{i}^{R}+S_{i}\right)+m_{i}\left(A_{i}^{W}+A_{i}^{C}\right)\right]\right\} \\
& \left\{\sum_{i} D_{i}\left[H_{i}^{W}+\left(H_{i}^{W}+H_{i}^{C}\right) / m_{i}-D_{i} \sum_{j} H_{i j} \lambda_{i j}{ }^{2}\right]\right\}
\end{aligned}
$$

We can obtain (33) for fixed $\lambda_{i j}$ :

$$
\begin{gathered}
\frac{d S_{E}\left(m_{i} / \lambda_{i j}\right)}{d m_{i}}=\left(A_{i}^{W}+A_{i}^{C}\right) \sum_{i} D_{i}\left(H_{i}^{W}-D_{i} \sum_{j} H_{i j} \lambda_{i j}{ }^{2}\right) \\
-\frac{\left(H_{i}^{W}+H_{i}^{C}\right) D_{i} \sum_{i}\left(A_{i}{ }^{R}+S_{i}\right)}{\sum_{i} m_{i}{ }^{2}} \\
\frac{d^{2} S_{E}\left(m_{i} / \lambda_{i j}\right)}{d^{2} m_{i}}=\frac{2\left(H_{i}^{W}+H_{i}{ }^{C}\right) D_{i} \sum_{i}\left(A_{i}^{R}+S_{i}\right)}{\sum_{i} m_{i}{ }^{3}}
\end{gathered}
$$

Since $d^{2} S_{E}\left(m_{i} / \lambda_{i j}\right) / d m_{i}^{2}>0$, we can obtain $m$ from $d S_{E} / d m_{i}=0$ and is given by

$$
m_{i}^{0}\left(\lambda_{i j}\right)=\left[\frac{\left(H_{i}^{W}+H_{i}^{C}\right) D_{i} \sum_{i}\left(A_{i}^{R}+S_{i}\right)}{\left(A_{i}^{W}+A_{i}^{C}\right) \sum_{i} D_{i}\left(H_{i}^{W}-D_{i} \sum_{j} H_{i j} \lambda_{i j}{ }^{2}\right)}\right]^{1 / 2}
$$

Substituting (35) into (31), we get $F\left(\lambda_{i j}\right)$ :

$$
\begin{aligned}
F\left(\lambda_{i j}\right)= & \left\{2 \sum_{i}\left(A_{i}^{R}+S_{i}\right)\left[\sum_{i} D_{i}\left(H_{i}^{W}-D_{i} \sum_{j} H_{i j} \lambda_{i j}{ }^{2}\right)\right]\right\}^{1 / 2} \\
& +\sum_{i}\left[2\left(A_{i}^{W}+A_{i}^{C}\right)\left(H_{i}^{W}+H_{i}^{C}\right) D_{i}\right]^{1 / 2}
\end{aligned}
$$

Since other terms in (36) are constant regardless of $\lambda_{i j}$ except $D_{i} \sum_{j} H_{i j} \lambda_{i j}{ }^{2}$, we reformulate the next problem equivalently as follows:

$$
\begin{gathered}
\text { Maximize } G_{i}\left(\lambda_{i}\right)=\sum_{j} H_{i j} \lambda_{i j}{ }^{2} \\
\text { s.t } \quad(27),(28),(29)
\end{gathered}
$$

This problem belongs to the class of quadratic maximization problems subject to linear constraints with a positive definite quadratic term. Since this problem aims to assign production allocation $\lambda i$, a heuristic procedure is proposed as follows to solve the model. The heuristics is

Step1. Resequence $H_{i j}$ in the descending order for product i, such that $H_{i 1} \geq H_{i 2} \geq H_{i 3} \geq \cdots \geq H_{i n}$;

Step2. $i=i+1$, Let $t$ be the current index number of the plant to be assigned, and

$$
R_{i t}=\sum_{j=1}^{t} \lambda_{i j}, t=0, R_{i t}=0
$$

Step3. $t=t+1$ assignment to production to the $t$ th plant point:

If $R_{i t}<1$ set

$$
\begin{aligned}
& \lambda_{i t}=\min \left\{1-R_{i t}, d_{i t} / D_{i}, Q_{i t} / D_{i} u_{i t}\right\} \\
& R_{i t}=R_{i, t-1}+\lambda_{i t} \text { go Step } 4
\end{aligned}
$$

Else $\lambda_{i t}=0, R_{i t}=R_{i, t-1}+\lambda_{i t}$

End if

Stetp4. if $t<n$, go to Step 3; else, go to Step5;

Step5. Calculate $G_{i}\left(\lambda_{i}\right)$;

Step6. if $i<m$, go Step 5 
Step7. Calculate $\sum_{i} D_{i}^{2} \sum_{j} H_{i j} \lambda_{i j}{ }^{2}$

Step8. Calculate $\mathrm{m}_{\mathrm{i}}^{*}$, if $\mathrm{m}_{\mathrm{i}}^{*}$ isn't interger, then

$$
\begin{aligned}
m^{*}=\arg _{m} \min \left\{F^{\prime} / m=\left(m_{1}, \ldots, m_{I}\right),\right. \\
\left.m_{i} \in\left\{\left[m_{i}^{0}\left(\wedge^{*}\right)\right],\left\lceil m_{i}^{0}\left(\wedge^{*}\right)\right]\right\}\right\}
\end{aligned}
$$

Else go Step 2

Step9. Calculate T*

Step10. Calculate F, stop

\section{Simulations and Performance Analysis}

To test the performance of the heuristics, some computation experimentations are conducted and their simulation results, as well as analysis, are presented in this section. The comparison of the heuristics and traditional quasiNewton method are reported and analyzed. To shorten the length of the paper and without loss of generality, this section presents an example with multiple products to illustrate the application of the model and the heuristics. For simplicity, multiple plants at the manufacturer are denoted by 1, 2, 3, they can all produce three products A, $\mathrm{B}$, C. The production rate, annual production capacity, Transfer rate and the holding cost are presented in Table 1. The production setup cost, the ordering cost and the holding cost at the manufacturer, the warehouse and the retailer and the demand rate are presented in Table 2.

Table 1. The parameters used in simulation tests

\begin{tabular}{ccccccc}
\hline \multirow{2}{*}{ Plants } & \multicolumn{3}{c}{ Production rate(unit) } & \multicolumn{3}{c}{ Annual Capacity(hours) } \\
\cline { 2 - 7 } & A & B & C & A & B & C \\
\hline 1 & 6000 & 7400 & 5000 & 0.2 & 0.35 & 0.45 \\
2 & 5700 & 11000 & 5800 & 0.2 & 0.35 & 0.45 \\
3 & 8000 & 10000 & 5700 & 0.2 & 0.35 & 0.45 \\
\hline \multirow{2}{*}{ Plants } & \multicolumn{2}{l}{ Transfer rate(unit/year) } & \multicolumn{2}{c}{ Holding cost(\$/unit) } \\
\cline { 2 - 7 } & A & B & C & A & B & C \\
\hline 1 & 3400 & 2800 & 3100 & 6 & 5 & 2 \\
2 & 3000 & 3200 & 3000 & 5 & 8 & 2 \\
3 & 4100 & 5200 & 3100 & 7 & 8 & 6 \\
\hline
\end{tabular}

Table 2. Basic data of the test

\begin{tabular}{lccc}
\hline \multicolumn{1}{c}{ costs } & A & B & C \\
\hline Setup cost (\$) & 600 & 500 & 200 \\
OR of raw material (\$) & 100 & 150 & 90 \\
Shipping cost(\$) & 25 & 20 & 20 \\
OR at the retailer $(\$)$ & 50 & 60 & 40 \\
HC of raw material $(\$)$ & 2 & 3 & 2 \\
HC at warehouse $(\$)$ & 8 & 8 & 8 \\
HC at the retailer $(\$)$ & 8 & 8 & 10 \\
Demand rate(unit) & 6000 & 7200 & 4300 \\
\hline
\end{tabular}

From Table 3, the production cycle length, delivery frequency in a cycle from the warehouse to the retailer, the production allocation of the two solutions with heuristics and QNM are compared. A near-optimal solution with relative deviation of $0.928 \%$ is obtained from the best solutions by QNM with feasible initial solution. Hence, one can conclude that the heuristics method is more effective than QNM in the case of the above example. In particular, the results have pointed out the significance of assigning production among the plants. It reveals that business operations, including production and distribution among the plants, should be considered in an integrative manner so as to reduce costs and enhance the enterprise's competitiveness.

To illustrate the effectiveness of the heuristics, four randomly generated examples with $3 * 5,5 * 5,5 * 10$, $10 * 10$ (plants*products) are cited to make the comparison between the heuristics and the QNM. From Table 4, one can see that the heuristic algorithm is better than the QNM in these examples in terms of quality of the solution.

\section{Conclusions}

One of the core problems of supply chain management is the coordination of production and distribution. This paper considers joint decisions in production cycle length, delivery frequency and production allocation for a single

\begin{tabular}{|c|c|c|c|c|c|}
\hline \multicolumn{3}{|c|}{ Delivery frequency } & \multicolumn{3}{|c|}{ Production allocation } \\
\hline & heuristics & QNM & & heuristics & QNM \\
\hline A & 6 & 6 & 1-A & 0.500 & 0.500 \\
\hline B & 5 & 5 & 1-B & 0.500 & 0.500 \\
\hline $\mathrm{C}$ & 5 & 5 & $1-\mathrm{C}$ & 0.000 & 0.000 \\
\hline \multirow{2}{*}{$\begin{array}{l}\text { Production } \\
\text { cycle }\end{array}$} & heuristics & 0.209 & 2-A & 0.389 & 0.301 \\
\hline & QNM & 0.207 & $2-B$ & 0.000 & 0.000 \\
\hline \multicolumn{3}{|c|}{ The total costs } & $2-\mathrm{C}$ & 0.611 & 0.699 \\
\hline \multicolumn{2}{|c|}{ heuristics } & 26705 & 3-A & 0.721 & 0.721 \\
\hline \multicolumn{2}{|c|}{ QNM } & 26953 & 3-B & 0.279 & 0.140 \\
\hline \multicolumn{2}{|c|}{ difference/\% } & 0.928 & $3-C$ & 0.000 & 0.139 \\
\hline
\end{tabular}

Table 3. Comparison of the heuristics and QNM

Table 4. Comparison of the heuristics and QNM for different size of examples

\begin{tabular}{cccc}
\hline \multirow{2}{*}{ Problem size } & \multicolumn{3}{c}{ Costs } \\
\cline { 2 - 4 } & heuristic & QNM & Diff (e/\%) \\
\hline $3 * 5$ & 26539 & 26916 & 1.42 \\
$5 * 5$ & 45339 & 46413 & 2.37 \\
$5 * 10$ & 45209 & 46520 & 2.89 \\
$10 * 10$ & 82336 & 83223 & 1.07 \\
\hline
\end{tabular}


product and for multiple products in a production- distribution network system with multiple plants and multiple retailers. All plants are all capacitated. Based on the production capacity and the unit production capacity for producing a product, the mathematical programming model is presented to distribute the demand of the retailer to multi-plants to achieve an objective of minimizing the average costs. Two effective heuristic methods are developed to solve the joint decision problem with single product and multiple products. The simulation results have shown that the heuristics is easily implemented and effective for the decision problems.

Future work includes: the economic allocation of the complex product in multiple plants.

\section{Acknowledgment}

The paper is financially supported by the Natural Science Foundation of China (NSFC 70625001, 70721001), National 973 program (2009CB320601) of China, and 111 project of Ministry of Education (MOE) in China with number B08015.

\section{REFERENCES}

[1] R. H. King and R. R. Love, "Coordinating decisions for increased profits,” Interfaces, Vol. 10, pp. 4-19, 1980.

[2] J. F. Williams, "Heuristic techniques for simultaneous scheduling of production and distribution multi-echelons structures: Theory and empirical comparisons,” Management Science, Vol. 27, pp. 336-352, 1981.

[3] R. M. Hill, “The single-manufacturer single-retailer integrated production-inventory model with a generalized policy,” European Journal of Operational Research, Vol. 97, pp. 493-499, 1997.

[4] I. David and M. Eben-Chaime, "How far should JIT vendor- buyer relationships go?” International Journal of
Production Economic, Vol. 81, pp. 361-368, 2003.

[5] T. Kim, Y. Hong, and J. Lee, "Joint economic production allocation and ordering policies in a supply chain consisting of multiple plants and a single retailer," International Journal of Production Research, Vol. 43, pp. 3619-3632, 2005.

[6] T. Kim and Y. Hong, “Technical note: Production allocation, lot-sizing, and shipment policies for multiple items in multiple production lines,” International Journal of Production Research, Vol. 46, pp. 289-294, 2008.

[7] R. W. Hall, "On the integration of production and distribution: Economic order and production quantity implications,” Transportation Research, Part B, Vol. 30, pp. 387-403, 1996.

[8] J. Hahm and C. A. Yano, "The economic lot and delivery scheduling problem: The single item case,” International Journal Production Econom, Vol. 28, pp. 238-252, 1992.

[9] J. Benjamin, “An analysis of inventory and transportation costs in a constrained network," Transportation Science, Vol. 23, pp. 177-183, 1989.

[10] D. E. Blumenfeld, L. D. Burns, and C. F. Daganzo, "Synchronizing production and transportation schedules," Transportation Research, Vol. 25, pp. 23-37, 1991.

[11] F. Fumero and C. Vercellis, "Synchronized development of production, inventory and distribution schedule," Transportation Science, Vol. 33, pp. 330-340, 1999.

[12] J. F. Tang, K. L. Yung, and A. W. H. Ip., "Heuristics based integrated decisions for logistics network systems," Journal Manufacturing Systems, Vol. 23 , pp. 1-13, 2004.

[13] K. L. Yung, J. F. Tang, A. W. H. Ip., and D. W. Wang, "Heuristics for joint decisions in production, transportation, and order quantity,” Transportation Science, Vol. 40, pp. 99-116, 2006.

[14] S. Sahni, “Computationally related problems," SIAM Journal on Computing, Vol. 3, pp. 262-279, 1974. 